\title{
TRATAMIENTO DE LA HIPERTENSIÓN Y EL IMPACTO DE LOS CAMBIOS EN EL ESTILO DE VIDA EN PACIENTES CON RIESGO CARDIOVASCULAR
}

\author{
Enrique Melgarejo Rojas, MD. Cardiólogi ${ }^{*}$, Diego Huertas Rosselli, MD. Pediatra ${ }^{2}$, \\ Luís Ernesto Rozo Maragua, MD. Internista ${ }^{3}$, Jorge Enrique Ospina Aguirre, MD, M.Sc. ${ }^{4} Y$ \\ Luis Atemo Gonzalez-Quevedo PS. ${ }^{5}$ \\ ${ }^{1}$ Cardiólogo-Internista, Electrofisiólogo, Universidad Militar Nueva Granada, Bogotá, Colombia. \\ ${ }^{2}$ Pediatra-Neumólogo, Bogotá, Colombia. ${ }^{3}$ Internista. México D.F. ${ }^{4}$ M.Sc. Farmacología, Bogotá, Colombia. \\ ${ }^{5}$ Psicólogo, especialista en epidemiología, Universidad Militar Nueva Granada, Bogotá, Colombia
}

\begin{abstract}
Resumen
La hipertensión arterial es un poderoso factor de riesgo cardiovascular y suele aparecer en asociación con otros factores que pueden empeorar su pronóstico y/o incrementar el riesgo cardiovascular. Por la literatura mundial se sabe que la modificación de los estilos de vida puede proveer reducciones adicionales en las cifras tensionales. El objetivo del presente estudio fue demostrar que empleando medicamentos anti-hipertensivos solos o en combinación buscando alcanzar las metas, y que adicionando unas recomendaciones de cambios de estilo de vida, ofrece un beneficio mayor en cuanto al impacto sobre un mejor logro de metas o reducción de la terapia farmacológica. Se hizo el seguimiento a 1.605 pacientes hipertensos obtenidos a través de la colaboración de 210 médicos de las ciudades más importante de Colombia durante cuatro meses, en un estudio de vida real, quienes fueron divididos en dos grupos; el primero o grupo control recibió la terapia convencional, y al criterio del médico, si fuese necesario, adicionar telmisartan 80 mg ó telmisartán + hidroclorotiazida (HCTZ) (en monoterapia de combinación) y el segundo o grupo balance, además del tratamiento se le adicionó una intervención conductual específica con recomendaciones puntuales para reducir el riesgo cardiovascular. Se evaluó en ambos casos las reducciones promedio de la Tensión Arterial (TA) sistólica y TA diastólica, así como el logro de las metas mediante la intervención adicional sobre cambio de estilo de vida. La reducción de la TA sistólica (promedio) obtenida fue de 26,1 mm Hg (DE 16,1) en el grupo balance y de 24,6 (DE 15.9) mm Hg en el grupo control. La reducción de TA diastólica fue de 13,98 (DE 9,1) mmHg y 13,37 (DE 9,1) mmHg para el grupo balance y el grupo control respectivamente. No se observaron cambios significativos entre los dos grupos, pero se alcanzaron las metas de cifras tensionales en 591 (75,7\%) del grupo control y en 635 (77\%) del grupo balance. Se concluye que telmisartán solo o asociado a HCTZ o a otras terapias antihipertensivas, fue efectivo en la reducción de las cifras tensionales tanto sistólica como diastólica, facilitando más el logro de metas. El grupo balance no mostró un resultado estadísticamente significativo, posiblemente a que se requiere mayor insistencia y periodicidad, y preferiblemente en forma personalizada la educación del paciente para lograr que cambie hacia un estilo de vida saludable que impacte significativamente en su tratamiento para lograr el objetivo de mayor reducción de las cifras tensionales.
\end{abstract}

Palabras clave: hipertensión, estilo de vida, educación, prevención de enfermedades, efectividad

\section{TREATMENT OF HYPERTENSION AND THE IMPACT OF LIFESTYLE CHANGES IN PATIENTS WITH CARDIOVASCULAR RISK}

\begin{abstract}
Arterial hypertension is a powerful factor of cardiovascular risk, and it usually appears in association with other factors that may worsen its diagnosis and/or increase cardio vascular risk. Through worldwide literature it is well known that a change of lifestyles can provide additional reductions of tensional figures. The main purpose of this study was to
\end{abstract}

Recibido: Mayo 18 de 2010 Aceptado: Marzo 9 de 2011

* Correspondencia: Enrique Melgarejo enrique.melgarejo@gmail.com. Dirección postal: Tr. 3 No 49-00. Facultad de Medicina, Universidad Militar Nueva Granada, Bogotá, Colombia. 
show that by using single or combined anti-hypertensive medications in order to reach objectives plus adding some recommendations for changes in the patients' lifestyles, a higher benefit is obtained as to the impact of the goals set or the reduction of the pharmacological therapy. Follow up of 1.605 hypertensive patients was carried out for four months, in a real-life study, thanks to the cooperation of 210 physicians from Colombia's leading cities. They were divided into two groups: the first, the control group, received conventional therapy and, according to the physician's criterion, if necessary, to add $80 \mathrm{mg}$ of telmisartan or telmisartan plus hydrochlorothiazide (HCTZ) (through combination and monotherapy); and the second, or balance group, in addition to the treatment, was given a concrete behavioral intervention through specific recommendations to reduce cardiovascular risk. Average reductions of Systolic Blood Pressure and Diastolic Blood Pressure were evaluated, as well as the achievement of set goals through the additional intervention of changes in lifestyles. The reduction obtained of the (average) systolic blood pressure was of $26.1 \mathrm{~mm}$ $\mathrm{Hg}$ (SD 16.1) in the balance group, and of 13.98 (SD 9.1) mm HG and 13.37 (SD 9.1) for the corresponding balance and control groups. No meaningful changes were observed between the two groups, but the goals regarding tensional figures in $591(75.7 \%)$ of the control group, and in $635(77 \%)$ of the balance group were achieved. It is concluded that telmisartan alone or in combination with HCTZ or other hypertensive therapies, was effective in the reduction of both systolic and diastolic tensional figures, thus facilitating the achievement of the set goals. The balance group did not show a meaningful statistical outcome, probably because a higher persistence and periodicity are required, and preferably under a personalized education given to the patients so that they change to a healthier lifestyle that has a meaningful impact on their treatment to achieve the objective of a greater reduction of tensional figures.

Key words: Hypertension, life style, diseases prevention, education, effectiveness

\title{
TRATAMENTO DA HIPERTENSÃO E O IMPACTO DAS MUDANÇAS NO MODO DE VIDA EM PACIENTES COM RISCO CARDIOVASCULAR
}

\begin{abstract}
Resumo
A hipertensão é um fator de risco cardiovascular poderosa e geralmente aparece em associação com outros fatores que podem piorar o prognóstico e / ou aumentar o risco cardiovascular. Literatura mundial é conhecido que a mudança de estilos de vida podem fornecer reduções adicionais da pressão arterial. $\mathrm{O}$ objetivo deste estudo foi demonstrar que o uso de medicamentos anti-hipertensivos sozinho ou em combinação para alcançar as metas, e acrescentando algumas recomendações para mudar de estilo de vida, fornece um benefício em termos de impacto sobre um melhor logro das metas o redução da terapia medicamentosa. Foi feito acompanhamento a 1.605 doentes hipertensos obtidos através da colaboração de médicos 210 das mais importantes cidades da Colômbia por quatro meses, em um estudo da vida real, foram divididos em dois grupos, o primeiro grupo ou controle recebeu terapia convencional segundo critério do médico, se necessário, adicionar telmisartan $80 \mathrm{mg}$ ou hidroclorotiazida + telmisartan (HCTZ) (em monoterapia em combinação) eo segundo grupo ou equilíbrio, além do tratamento foi adicionado uma intervenção comportamental específica para recomendações específicas para reduzir o risco cardiovascular. Foi avaliada em ambos os casos as reduções médias na pressão arterial (PA) sistólica e pressão arterial (PA) diastólica, assím como o logro a das metas por meio de intervenção adicionais sobre a mudança de estilo de vida. A redução da pressão arterial diastólica foi 13,98 (DP 9,1) mmHg e 13,37 (SD 9,1) mmHg para o grupo de equilíbrio e grupo controle, respectivamente. Não foram observadas alterações significativas entre os dois grupos, mas as metas para a pressão arterial em 591 (75,7\%) do grupo controle e $635(77 \%)$ na balança. Concluímos que a telmisartan em monoterapia ou associado a HCTZ ou outro terapia anti-hipertensiva foi eficaz na redução da pressão arterial sistólica e diastólica, tornando mais fácil para alcançar metas. O grupo de equilíbrio não mostrou resultado estatisticamente significativa, possivelmente é necessária ênfase maior e freqüência e, de preferência em uma educação individual do doente para alcançar a mudança em direção a um estilo de vida saudável que irá impactar de forma significativa em seu tratamento para atingir o objectivo de uma maior redução da pressão arterial.
\end{abstract}

Palavras-chave: : hipertensão, estilo de vida, educação, prevenção de doenças, efetividade 


\section{Introducción}

La enfermedad cardiovascular es una de las enfermedades crónicas con altos índices de mortalidad y morbilidad a nivel mundial y varios estudios han mostrado que tanto el estilo de vida como los patrones de conducta son unos de los factores que contribuyen con estos índices. En Colombia la mortalidad por enfermedad cardiovascular es del $28 \%$ siendo la segunda causa generadora de enfermedad y la primera causa de pérdida de años de vida saludable (AVISA); la prevalencia de hipertensión en algunas zonas urbanas es de $14,1 \%(1,2)$ y la mortalidad de la enfermedad hipertensiva es de $9,7 \%$ en hombres y $12,2 \%$ en mujeres, mientras que la de la enfermedad cardiovascular general es de $23,4 \%$ y $33,5 \%$ respectivamente. La tasa estandarizada de muerte por enfermedad hipertensiva (en 1999) era de 27,59 en hombres y de 27,34 en mujeres por cada 100.000 (3). La prevalencia de la hipertensión arterial ajustada por edad aumenta con los años, de forma similar a lo que se observa en la población mundial.

La hipertensión arterial (HTA) se asocia con la industrialización, las migraciones, a la herencia (primer grado de consanguinidad) y con características psicosociales tales como el ingreso económico, el hábitat y los rasgos de personalidad. Sin embargo, a la luz de la evidencia actual, la HTA no debe verse simplemente como un Factor de Riesgo (FdeR) de eventos cardiovasculares, puesto que al igual de otros denominados como FdeR (diabetes, dislipidemia, tabaquismo etc), cuando la persona lo presenta o está expuesta, ya hay daño vascular, o por lo menos, disfunción endotelial demostrada -entre otros métodos- por la alteración de la vasodilataciòn mediada por flujo. De igual forma, el hipertenso no tratado y/o no controlado, ya tiene -tempranamente- sobre-expresado el sistema adrenérgico y el sistema renina-angiotensina, tanto el circulante como el tisular (y probablemente el intracrino o celular), con el impacto que esta situación conlleva en cuanto a morbi-mortalidad por disfunción precoz (y muchas veces irreversible) de los órganos blanco.

Aunado a lo anterior, la HTA no suele ocurrir como un fenómeno aislado sino que con mucha frecuencia los pacientes hipertensos presentan concomitantemente otros factores adicionales de riesgo, que en conjunto incrementan las posibilidades de ocurrencia de desenlaces tales como accidentes cerebro vasculares, enfermedad coronaria en sus diferentes manifestaciones (muerte súbita, infarto, angina inestable o falla cardiaca) y de enfermedad arterial periférica (4-7).

Existe información suficiente en la literatura médica para suponer que el tratamiento farmacológico como única inter- vención terapéutica puede no ser la única medida eficaz para alcanzar las metas de presión arterial y para reducir el riesgo cardiovascular global (8-10) y que es por lo tanto necesaria la instauración concomitante de cambios en el estilo de vida como primera intervención médica, que de hecho dichos cambios, representan la piedra angular de todas las guías de tratamiento de la hipertensión $(11,12)$. Particularmente los cambios en el estilo de vida deben aplicarse como terapia inicial en pacientes hipertensos en el estadio I de la enfermedad, facilitándose además la disminución de la medicación o su retiro completo en los pacientes hipertensos bien controlados y debe también aplicarse como pivote fundamental en la población hipertensa de alto riesgo (12).

Entre las modificaciones del estilo de vida (hoy día mejor denominados como cambios terapéuticos en el estilo de vida) que han demostrado reducir la presión arterial se incluyen la disminución de la ingesta de sodio, la reducción del sobrepeso corporal, especialmente en el hipertenso con sobrepeso $\mathrm{u}$ obesidad androide (por predominio de la grasa abdominal, la cual induce inflamación vascular y resistencia a la insulina, entre otras acciones), la moderación de la ingesta de alcohol y fundamentalmente el aumento de la actividad física. También debe recomendarse la instauración de una dieta rica en frutas y vegetales (dieta DASH), así como la reducción del consumo de alimentos con alto contenido de grasas saturadas y trans (11).

Realmente, el ejercicio es el "medicamento" con mayor relación en cuanto a costo-efectividad para disminuir cifras tensionales. El hecho de bajar de peso con el ejercicio, ayuda a corregir la disglucemia y dislipidemias (incluso incrementa la fracción HDL del colesterol), además de estimular la producción del óxido nítrico con todos sus beneficios que esto implica, mejorar también el estado anímico y la calidad de vida en la vejez.

Sobre la eficacia de los cambios en el estilo de vida para reducir las cifras de tensión arterial, el estudio clínico realizado por Whelton y col. (12) en una población de 975 pacientes hipertensos (hombres y mujeres) entre los $60 \mathrm{y}$ los 80 años y a los que se les hizo un seguimiento hasta de 30 meses, demostró que la reducción en la ingesta de sodio y la reducción del peso corporal fueron eficaces para el control de la hipertensión. El 30\% de los pacientes lograron suspender la medicación y el grupo de pacientes que disminuyeron de peso y adoptaron la dieta hiposódica requirieron solo la mitad de las cantidades previas de medicamentos antihipertensivos. 
En el estudio de tipo experimental realizado por Appel y col. (13) en el cual participaron 810 pacientes adultos quienes hicieron un cambio de estilo de vida a través de intervenciones conductuales, se demostró que sí es posible por medio de estos cambios reducir las cifras de tensión arterial, controlar mejor la hipertensión arterial, disminuir la prevalencia de hipertensión en pacientes de mediana edad y que sus beneficios son extensivos a pacientes que genéticamente estén en riesgo de desarrollar hipertensión arterial. En consecuencia, este estudio mostró que los cambios en el estilo de vida implementados bajo situaciones ideales son muy eficaces para reducir las cifras tensionales; sin embargo, la realización óptima de estas intervenciones (18 sesiones de consejería especializada durante un periodo de seis meses) suele ser de difícil ejecución en la práctica clínica habitual dentro del sistema de salud vigente en el país, y queda demostrada la importancia de esta intervención adicional a la farmacoterapia.

El control eficaz de las cifras tensionales fundamentalmente de variables como la adherencia del paciente al tratamiento farmacológico, el uso de una sola toma diaria la facilita (hecho que se logró significativamente en el presente estudio), de la escogencia de medicamentos que logren la presencia de niveles plasmáticos suficientes a lo largo de las 24 horas del día mediante una vida media o volumen de distribución que aseguren mediante su farmacocinética y farmacodinamia un estado de equilibrio óptimo y temprano para, por un lado mantener en lo posible el control de las cifras tensionales durante las 24 horas, y por el otro, evitar los picos o excursiones hipertensivas, los cuales magnifican el daño endotelial. Si se logra controlar las variables mencionadas, se puede reducir considerablemente la morbi-mortalidad cardiovascular atribuible a la enfermedad hipertensiva, incluso en los enfermos multi-riesgo (14-16).

El presente estudio estuvo dirigido a documentar las reducciones en las cifras tensionales alcanzadas por una cohorte de pacientes tratados con telmisartan o telmisar$\tan +$ hidroclorotiazida -de acuerdo a las consideraciones anteriormente expuestas- luego de cutaro meses de tratamiento, en pacientes hipertensos con o sin tratamiento previo antihipertensivo, y describir la magnitud de las diferencias alcanzadas en la reducción de cifras tensionales cuando a un grupo (grupo control) se les hace las recomendaciones habituales, frente a otro grupo (grupo balance), al cual se le adicionó un programa de intervención conductual para modificar el estilo de vida mediante una charla multidisciplinaria y seguimiento telefónico quincenal.

\section{Metodología}

Fue un estudio observacional, descriptivo y multicéntrico. Se hicieron tres consultas y registros de los cambios en las cifras tensionales de los pacientes de ambos grupos, durante cuatro meses de tratamiento (gráfica 1).

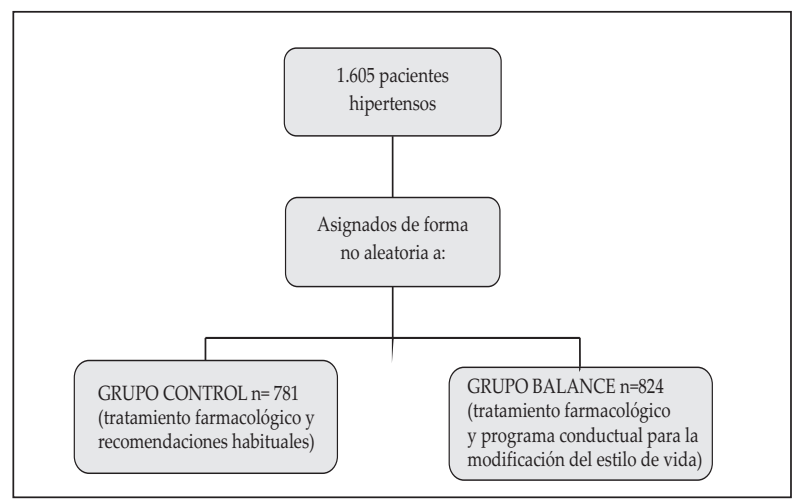

Gráfica 1. Perfil general del estudio

\section{Población de estudio y diseño de muestra}

Se trata del primer trabajo realizado a nivel nacional para determinar si unas recomendaciones de cambios de estilos de vida saludable en una población hipertensa, mejora el impacto que tienen los medicamentos sobre la hipertensión. Se seleccionó una muestra obtenida a través de la colaboración de 210 médicos generales y especialistas, de 1605 pacientes (hombres y mujeres) hipertensos mayores de 18 años de las principales ciudades de Colombia, con o sin tratamiento previo y que mediante firma del consentimiento informado, aceptaron su inclusión en el estudio. Los pacientes se dividieron en dos grupos y se les administró un medicamento (combinado o no con diurético) de reconocida eficacia por su farmacocinética y farmacodinamia y evidencia clínica, adicionándolo a otros fármacos previamente utilizados para el manejo de la HTA, en enfermos que aún no habían logrado las metas, o utilizándolo como terapia de inicio en enfermos hipertensos nuevos y sin tratamiento previo.

\section{Tipo de muestreo empleado}

Muestreo no probabilístico. Por tratarse de un estudio observacional de condiciones de vida real, no hubo una asignación de tratamientos de forma aleatorizada sino que cada médico tratante evaluó de forma independiente cada caso por separado y después de consultarlo con el paciente (interés por cambiar sus hábitos, disponibilidad de tiempo, 
etc), tomó la decisión de inscribirlo en el grupo control o en el grupo balance. Los tratamientos farmacológicos recibidos por los pacientes están resumidos en la tabla 1. Cada profesional vinculó entre cinco y diez pacientes

Tabla 1. Tratamientos farmacológicos por grupo

\begin{tabular}{lcc}
\hline $\begin{array}{c}\text { Medicamento administrado } \\
\text { una vez al día }\end{array}$ & $\begin{array}{c}\text { Grupo } \\
\text { CONTROL } \\
\mathbf{n}=781\end{array}$ & $\begin{array}{c}\text { Grupo } \\
\text { BALANCE } \\
\mathbf{n}=824\end{array}$ \\
\hline $\begin{array}{l}\text { Telmisartan } 80 \mathrm{mg} \\
\text { Telmisartan } 80 \mathrm{mg}+\end{array}$ & $390(47,4 \%)$ & $432(52,6 \%)$ \\
$\begin{array}{l}\text { Hidroclorotiazida } 12.5 \mathrm{mg} \\
\text { Telmisartan } 80 \mathrm{mg}+\end{array}$ & $305(50,4)$ & $300(49,6)$ \\
Hidroclorotiazida $25 \mathrm{mg}$ & $86(48,3 \%)$ & $92(51,7 \%)$ \\
\hline
\end{tabular}

Nota: Los datos están expresados en forma de número de pacientes (valor porcentual).

Los grupos -no aleatorizados sino hechos según criterio médico (de acuerdo al grado de severidad de la enfermedad hipertensiva) y/o disponibilidad por parte del paciente, se distribuyeron así: grupo control, $\mathrm{n}=781$, terapia farmacológica + recomendaciones habituales sobre adopción de cambios en el estilo de vida. Grupo balance, $n=824$, terapia farmacológica + recomendaciones habituales sobre adopción de cambios en el estilo de vida + inclusión del paciente en un programa de intervención conductual de cuatro meses de duración con miras a modificar su estilo de vida. El programa de intervención conductual consistió en una charla educativa de 30 minutos desarrollada por un grupo interdisciplinario, se les entregó un material escrito de tipo informativo y se realizó seguimiento telefónico quincenal durante los cuatro meses del estudio para reforzar la motivación de tener estilos y hábitos de vida saludable (reducción de sobrepeso, reducción de la ingesta de sodio, cesación del tabaquismo, aumento de la actividad física, implementación de la dieta DASH y reducción de la ingesta de alcohol).

\section{Resultados}

El promedio de las cifras tensionales al inicio del estudio fue de $147 / 93 \mathrm{mmHg}$ en pacientes que venían recibiendo medicación convencional, o enfermos diagnosticados como hipertensos nuevos sin tratamiento previo, a quienes se les administró al inicio o se les adicionó al tratamiento telmisartán $80 \mathrm{mg}$ y si no respondían en cuanto al logro de metas, se adicionó telmisartán + hidroclorotiazida en una sola tableta $12,5 \mathrm{mg}$ hasta $25 \mathrm{mg}$.

La edad promedio de los pacientes participantes en el estudio fue de 62 años con desviación típica 13,2; el 43\% eran hombres y el 57\% mujeres. El $49 \%$ de los pacientes reci-

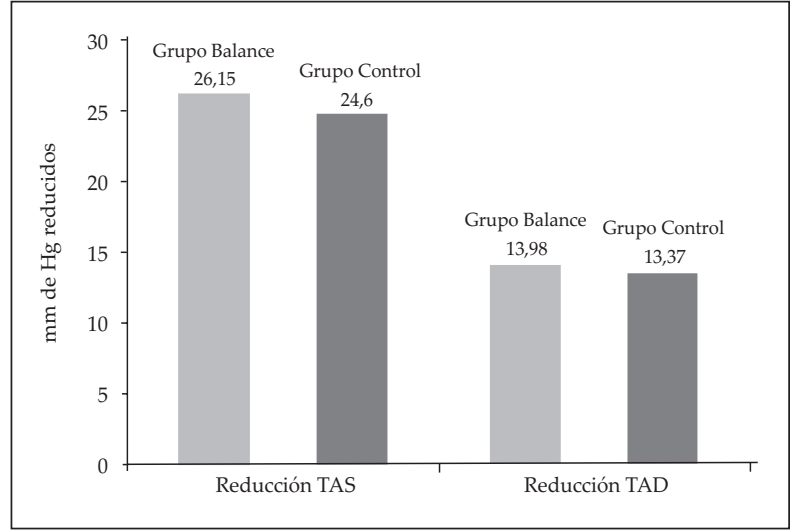

Gráfica 2. Reducciones alcanzadas en las cifras tensionales por grupo

bieron telmisartán o telmisartán + HCTZ y el 51\% restante además del medicamento anterior recibieron orientaciones de hábitos saludables de estilo de vida adicionales.

La reducción promedio de las cifras tensionales para toda la población en estudio fue de $25,35 \mathrm{mmHg}$ para tensión sistólica (DE 16,18) y de 16,8 mmHg para tensión diastólica (DE 9,3). La reducción en las cifras de TA sistólica en el grupo balance fue de $26,1 \mathrm{mmHg}$ (DE 16,1) y en el grupo control fue de 24,6mmHg (DE 15.9) mmHg. La reducción en las cifras de TA diastólica en el grupo balance fue de 13,98 (DE 9,1) mmHg y 13,37mmHg en el grupo control (DE 9,1) (gráfica 2).

La población del estudio tenía un riesgo cardiovascular importante (tabaquismo, predominio de HTA sistólica, dislipidemia, enfermedad coronaria y arterial periférica, antecedentes familiares, y un número significativo de diabéticos (Tabla 2). La mayoría de los pacientes (más de la mitad) no recibían tratamiento anti-hipertensivo previo. Los medicamentos más utilizados por los pacientes que recibía tratamiento (antes de adicionar Telmisartán) fueron IECAS, seguidos de calcioantagonistas, betabloqueadores y por último diuréticos

De los 1605 pacientes, el control de las cifras tensionales (cifras menores a $140 / 90 \mathrm{mmHg}$ ) fue alcanzado en 1.226 pacientes $(76,38 \%)$, de los cuales 591 (75,7\%) pertenecía al grupo control y 635 (77\%) al grupo balance.

\section{Discusión}

Debido a las limitaciones propias del diseño de un estudio observacional como la no asignación al azar de los grupos y los diferentes estadios de la enfermedad al inicio de la observación, este tiene únicamente un alcance descriptivo 
Tabla 2. Características de la muestra

\begin{tabular}{|c|c|c|}
\hline $\begin{array}{c}\text { 1. Características clínicas } \\
\text { y demográficas }\end{array}$ & $\begin{array}{c}\text { Grupo } \\
\text { CONTROL } \\
n=781\end{array}$ & $\begin{array}{c}\text { Grupo } \\
\text { BALANCE } \\
n=824\end{array}$ \\
\hline Hombres & $342(43,8 \%)$ & $343(41,6 \%)$ \\
\hline Mujeres & $439(56,2 \%)$ & $481(58,4 \%)$ \\
\hline Edad (años) & $61,6(12,6)$ & $61,5(12,5)$ \\
\hline$\leq 60$ años & $421(54 \%)$ & $442(53,6 \%)$ \\
\hline$>60$ años & $360(46 \%)$ & $382(46,4 \%)$ \\
\hline Tabaquismo & $168(21,5 \%)$ & $146(17,7 \%)$ \\
\hline Presión arterial diastólica (mmHg) & $93,3(11,9)$ & $93,2(11,7)$ \\
\hline Presión arterial sistólica (mmHg) & $154(20)$ & $153(19,8)$ \\
\hline Frecuencia cardiaca por minuto & $78(8,9)$ & $77,3(8,9)$ \\
\hline $\mathrm{IMC}\left(\mathrm{Kg} / \mathrm{m}^{2}\right)$ & $27,5(4,2)$ & $27,4(4,2)$ \\
\hline Peso corporal (Kg) & $73,5(13,3)$ & $72,8(13,3)$ \\
\hline Colesterol LDL (mg/dL) & $156(58,8)$ & $146(58,7)$ \\
\hline Colesterol HDL (mg/dL) & $47,6(30,8)$ & $47,7(30,8)$ \\
\hline Triglicéridos (mg/dL) & $195,5(89,2)$ & $195,6(89,3)$ \\
\hline Glicemia en ayunas (mg/dL) & $106(43,6)$ & $106,5(43,5)$ \\
\hline \multicolumn{3}{|l|}{ 2. Antecedentes médicos } \\
\hline \multirow{5}{*}{$\begin{array}{l}\text { Enfermedad coronaria } \\
\text { Enfermedad cerebrovascular } \\
\text { Enfermedad arterial periférica } \\
\text { Diabetes Mellitus } \\
\text { Historia familiar de enfermedad } \\
\text { cardiovascular }\end{array}$} & $106(13,6 \%)$ & $97(11,8 \%)$ \\
\hline & $32(4,1 \%)$ & $30(3,6 \%)$ \\
\hline & $131(16,8 \%)$ & $111(13,5 \%)$ \\
\hline & $167(21,4 \%)$ & $131(15,9 \%)$ \\
\hline & $461(59 \%)$ & $493(59,8 \%)$ \\
\hline \multicolumn{3}{|l|}{ 3. Terapia farmacológica actual } \\
\hline Ninguna & $479(61,3 \%)$ & $472(57,3 \%)$ \\
\hline Bloqueadores $\beta$ adrenérgicos & $51(6,5 \%)$ & $62(7,5 \%)$ \\
\hline Bloqueadores de canales de calcio & $61(7,8 \%)$ & $67(8,1 \%)$ \\
\hline Diuréticos & $30(3,8 \%)$ & $22(2,7 \%)$ \\
\hline Inhibidores de la ECA & $132(16,9 \%)$ & $148(18 \%)$ \\
\hline Otros & $28(3,6 \%)$ & $53(6,4 \%)$ \\
\hline
\end{tabular}

Nota: Los datos están expresados en forma de cantidad

(valor porcentual) o promedio (desviación estándar).

y no resulta posible ni apropiado hacer inferencias de tipo estadístico hacia todo el universo de pacientes hipertensos colombianos; sin embargo, hace que la población y el escenario del estudio (la práctica clínica cotidiana) describan la realidad de los pacientes que asisten al consultorio del clínico colombiano.

Llama la atención que aunque las reducciones en las cifras tensionales y la proporción de pacientes que alcanzaron las metas de sus cifras tensionales fue mayor en el grupo balance, la diferencia observada con el grupo control es muy escasa y estadísticamente no es significativa, hallazgo que es diferente a los reportados en otros estudios experimentales $(8,13)$; una explicación posible es que la intensidad, la duración, la frecuencia y el seguimiento periódico de la intervención realizada en el grupo balance no es equiparable a las realizadas en dichos estudios $(8,13)$. Varios reportes han mostrado la efectividad de las intervenciones no farmacológicas para generar reducciones en las cifras tensionales adicionales a las generadas por la terapia con medicamentos. Se puede concluir que aunque la modificación de estilos de vida es una medida eficaz, esta debe obedecer a políticas institucionales intensivas, frecuentes, y sostenidas en el tiempo, que formen parte de un plan de educación para la población en general y que el reforzamiento de esta sea preferiblemente de manera personal y no por vía telefónica.

Como fortalezas de este diseño podemos señalar el tamaño de la población observada, equivalente a dos veces la población del estudio PREMIER, (8) así como el hecho de haber realizado las observaciones y análisis en las diez principales ciudades del país en situaciones de vida "real". También se pudo corroborar los resultados obtenidos por otros autores en múltiples estudios a nivel internacional sobre la utilidad de telmisartan y especialmente su impacto en la reducción y control de las cifras de TA sistólica, y su utilidad en enfermos multi-riesgo como fue demostrado en el estudio ONTARGET (17).

\section{Conclusiones}

El manejo de la enfermedad hipertensiva con telmisartán con o sin diurético, como monoterapia o asociado a otros medicamentos antihipertensivos, fue eficaz en la reducción de las cifras tensionales, llegándose a un logro de metas en un $76 \%$,.y con mayor impacto en las cifras de TA sistólica.

Queda demostrado que para que un paciente hipertenso cambie sus hábitos de vida hacia unas recomendaciones saludables requiere de una educación persistente para que incorpore racionalmente el mensaje sobre la importancia que tiene la prevención en la salud.

Se espera que los resultados de este estudio observacional motive la realización de nuevos estudios a nivel nacional, al Sistema de Salud y a las diferentes EPS y aseguradoras, y a los médicos para que implementen de una manera persistente y reiterativa las estrategias para lograr los cambios en el estilo de vida en la población, y que tomen conciencia que esta es una medida crucial para la reducción de las cifras tensionales y del riesgo cardiovascular global. Definitivamente la prevención es y deberá ser siempre el primer acto médico que emplemos frente a una persona que nos consulte 


\section{Agradecimientos}

A Laboratorio Boheringer Ingelhaim Colombia por facilitar el medicamento Micardis ${ }^{\circledR}$. A Elizabeth Cornejo Laboratorio Novartis por su apoyo bibliográfico.

\section{Referencias}

1. Fajardo H.A. Prevalencia factores de riesgo cardiovascular. Localidad de los Mártires. Bogotá - Colombia [Internet]. [cited 2009 Mar 11 ] Available from: www.revmed.unal.edu.co/revistafm/v51n4/ v51n4pdf/v51n4a3.pdf

2. Norma técnica para la Prevención de enfermedad crónica y mantenimiento de la salud en el individuo sano mayor de 45 años [Internet]. [cited 2009 Mar 11 ] Available from: http://74.125.47.132/ search?q=cache:T318vnYruYMJ:www.minproteccionsocial. gov.co/vbecontent/library/documents/DocNewsNo16062DocumentNo4072.PDF+Prevalencia+factores+de+riesgo+cardiovascular. + Localidad+de+los+M\%C3\%A1rtires+Bogot $\% \mathrm{C} 3 \% \mathrm{~A} 1 \& \mathrm{hl}=\mathrm{es} \& \mathrm{ct}=\mathrm{cl}$ nk\&cd $=4 \& g \mathrm{l}=\mathrm{co} \& \mathrm{client}=$ firefox-a

3. Cubillos-Garzón LA, Casas JP, Morillo CA, Bautista LE. Congestive heart failure in Latin America: the next epidemic. Am Heart J 2004; 147:412-417

4. Ebrahim S, Montaner D, Lawlor DA. Clustering of risk factors and social class in childhood and adulthood in British women's heart and health study: cross sectional analysis. BMJ. 2004 Apr 10;328(7444):861.

5. Power C, Atherton K, Manor O. Co-occurrence of risk factors for cardiovascular disease by social class: 1958 British birth cohort. J Epidemiol Community Health. 2008 Dec ;62(12):1030-5.

6. Hae Guen Song, Eung Ju Kim, Hong Seog Seo, Seong Hwan Kim, Chang Gyu Park, Seong Woo Han, Ryu K. Relative contributions of different cardiovascular risk factors to significant arterial stiffness [Internet]. Int J Cardiol. 2008 Nov 26; [cited 2009 Mar 12 ] Available from: http://www.ncbi.nlm.nih.gov/pubmed/19041146

7. Collins AJ, Vassalotti JA, Wang C, Li S, Gilbertson DT, Liu J, Foley RN, Chen S, Arneson TJ. Who should be targeted for CKD screening? Impact of diabetes, hypertension, and cardiovascular disease. Am J Kidney Dis. 2009 Mar ;53(3 Suppl 3):S71-7.

8. Gordon NF, Salmon RD, Franklin BA, Sperling LS, Hall L, Leighton RF, Haskell WL. Effectiveness of therapeutic lifestyle changes in patients with hypertension, hyperlipidemia, and/or hyperglycemia. Am J Cardiol. 2004 Dec 15;94(12):1558-61

9. Appel LJ, Champagne CM, Harsha DW, Cooper LS, Obarzanek E, Elmer PJ, Stevens VJ, Vollmer WM, Lin P, Svetkey LP, Stedman SW, Young DR. Effects of comprehensive lifestyle modification on blood pressure control: main results of the PREMIER clinical trial. JAMA. 289(16):2083-93.

10. Mulrow CD, Chiquette E, Angel L, Grimm R, Cornell J, Summerbell $\mathrm{CD}$, Anagnostelis BB, Brand M. WITHDRAWN: Dieting to reduce body weight for controlling hypertension in adults. Cochrane Database Syst Rev. 2008 ;(4):CD000484.

11. Chobanian AV, Bakris GL, Black HR, Cushman WC, Green LA, Izzo JL, Jones DW, Materson BJ, Oparil S, Wright JT, Roccella EJ. The Seventh Report of the Joint National Committee on Prevention, Detection, Evaluation, and Treatment of High Blood Pressure: The JNC 7 Report. JAMA. 2003 May 14;289.19.2560.

12. Mancia G, De Backer G, Dominiczak A, Cifkova R, Fagard R, Germano G, Grassi G, Heagerty AM, Kjeldsen SE, Laurent S, Narkiewicz K, Ruilope L, Rynkiewicz A, Schmieder RE, Boudier HAS, Zanchetti A. 2007 ESH-ESC Practice Guidelines for the Management of Arterial Hypertension: ESH-ESC Task Force on the Management of Arterial Hypertension. J Hypertens. 2007 Sep 25(9):1751-62

13. Appel LJ. Nonpharmacologic therapies that reduce blood pressure: a fresh perspective. Clin Cardiol. 1999 Jul ;22(7 Suppl):III1-5.

14. Whelton PK, Appel LJ, Espeland MA, Applegate WB, Ettinger WH, Kostis JB, Kumanyika S, Lacy CR, Johnson KC, Folmar S, Cutler JA. Sodium reduction and weight loss in the treatment of hypertension in older persons: a randomized controlled trial of nonpharmacologic interventions in the elderly (TONE). TONE Collaborative Research Group. JAMA. 1998 Mar 18;279(11):839-846.

15. Gosse $\mathrm{P}$, Neutel JM, Schumacher $\mathrm{H}$, Lacourcière $\mathrm{Y}$, Williams B, Davidai $\mathrm{G}$. The effect of telmisartan and ramipril on early morning blood pressure surge: a pooled analysis of two randomized clinical trials. Blood Press Monit. 2007 Jun ;12(3):141-7.

16. Unger $\mathrm{T}$. The ongoing telmisartan alone and in combination with ramipril global endpoint trial program. Am J Cardiol. 2003 May 22;91(10A):28G-34G.

17. (Telmisartan, Ramipril, or Both in Patients at High Risk for Vascular EventsN The ONTARGET Investigators. N Engl J Med 2008; 358:1547-1559April 10,2008 